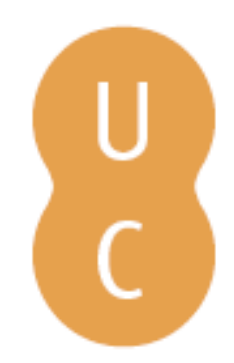

\title{
nombalina
}

\section{El linaje de Eva: revisiones de mitos clásicos en las poetas españolas bajo el franquismo}

\author{
Autor(es): $\quad$ Alonso Valero, Encarna \\ Publicado por: Imprensa da Universidade de Coimbra \\ URL \\ persistente: URI:http://hdl.handle.net/10316.2/44008 \\ DOI: $\quad$ DOl:https://doi.org/10.14195/978-989-26-1550-9_6 \\ Accessed : $\quad$ 26-Apr-2023 12:08:25
}

A navegação consulta e descarregamento dos títulos inseridos nas Bibliotecas Digitais UC Digitalis, UC Pombalina e UC Impactum, pressupõem a aceitação plena e sem reservas dos Termos e Condições de Uso destas Bibliotecas Digitais, disponíveis em https://digitalis.uc.pt/pt-pt/termos.

Conforme exposto nos referidos Termos e Condições de Uso, o descarregamento de títulos de acesso restrito requer uma licença válida de autorização devendo o utilizador aceder ao(s) documento(s) a partir de um endereço de IP da instituição detentora da supramencionada licença.

Ao utilizador é apenas permitido o descarregamento para uso pessoal, pelo que o emprego do(s) título(s) descarregado(s) para outro fim, designadamente comercial, carece de autorização do respetivo autor ou editor da obra.

Na medida em que todas as obras da UC Digitalis se encontram protegidas pelo Código do Direito de Autor e Direitos Conexos e demais legislação aplicável, toda a cópia, parcial ou total, deste documento, nos casos em que é legalmente admitida, deverá conter ou fazer-se acompanhar por este aviso.

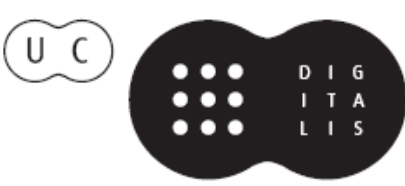




\section{Desplazamientos de la tradición clásica en las culturas hispánicas}

Paola Bellomi, Claudio Castro Filho, Elisa Sartor (eds.)

IMPRENSA DA UNIVERSIDADE DE COIMBRA 


\title{
El linaje de Eva: revisiones de mitos clásicos EN LAS POETAS ESPAÑOLAS BAJO EL FRANQUISMO ${ }^{1}$ (The lineage of Eve: revisions of classical myths in Spanish women poets under Franco's regime)
}

\author{
Encarna Alonso Valero \\ Universidad de Granada (Orcid: 0000-0002-3947-0304)
}

\begin{abstract}
Resumen: En 1947 se publicó Mujer sin Edén, de Carmen Conde, que fue el detonante de un fructífero diálogo poético entre un gran número de poetas de posguerra, que se interpelaban en sus poemarios. Entre esas respuestas se encuentra el famoso Mujer de barro de Ángela Figuera, que reescribe los más importantes arquetipos bíblicos femeninos (Eva, María, Sara, etc.) para, de ese modo, establecer una línea de género dentro de la poesía social bajo el franquismo. Esa cadena, en diálogo, de reescrituras subversivas de mitos clásicos femeninos va a permitir nuevas lecturas que muestran construcciones hasta ese momento inéditas de activos sujetos femeninos que contestan el ideario tradicional de la mujer como objeto y en particular como objeto de la poesía. Esta tendencia, además, no se agotó con el franquismo, sino que se consolidó en los años ochenta. Con frecuencia, ese nuevo sujeto activo toma en esos momentos la forma de un sujeto erótico que, con un erotismo lúdico, utiliza la subversión, la ironía y la parodia para encontrar un nuevo modo de decir el cuerpo, la sexualidad y los sentimientos femeninos. Dentro de ese objetivo, la frecuente revisión y subversión de mitos clásicos tiene una presencia fundamental.
\end{abstract}

Palabras clave: campo literario, poetas mujeres, posguerra, arquetipos bíblicos femeninos.

Aвstract: Published in 1947, Mujer sin Edén by Carmen Conde triggered a fruitful poetic dialogue amongst a great number of post-war poets, who started a conversation through their poetry books. Among the responses it generated, Mujer de barro by Ángela Figuera rewrites the most important feminine biblical archetypes (Eve, Mary, Sarah, etc.) so as to establish a gender line within social poetry during the Franco dictatorship. This chain of dialoguing subversive rewritings of feminine classical myths promoted new readings, which showed previously unknown constructs by active feminine subjects who rejected the traditional representation of women as objects and, especially, as objects of poetry. This trend did not fade out with the end of the dictatorship, quite the contrary, it was consolidated in the 1980s. This new active subject often appears as an erotic one who playfully uses subversion, irony and parody to find a new way to talk about the body, sexuality and women's feelings.

KEYWORDs: literary field, women poets, post-war, feminine biblical archetypes.

${ }^{1}$ Este trabajo forma parte de los resultados del proyecto de I+D La configuración del patrón poético español tras la Guerra Civil: relaciones literarias, culturales y sociales (FFI2013-44041-P), financiado por el Ministerio de Economía y Competitividad y FEDER. 
En este trabajo nos proponemos reflexionar acerca de cómo reescribir (o, en ocasiones, subvertir) mitos clásicos femeninos era para las poetas españolas bajo el franquismo una forma efectiva de hablar de problemas de su presente pero burlando o disminuyendo la presión de la censura. Aludir a mitos clásicos, como hablar de tiempos o personajes pasados, era uno de los recursos para poder sacar al espacio público discursos que, hechos explícitos, el régimen no toleraría, o al menos no tan fácilmente.

Esas reescrituras de mitos clásicos tienen otra consecuencia importante: provocan una redefinición de la experiencia poética y de las realidades susceptibles de ser poetizadas que da lugar a nuevas formas de lenguaje y de expresión. Cuando el orden aparece desafiado por un grupo, aunque sea con todas las limitaciones que impone escribir y publicar bajo una dictadura, la experiencia hasta entonces no hablada se encuentra bruscamente expresada en público. Así, las experiencias privadas sufren un cambio de estado cuando se reconocen a sí mismas en la objetividad pública de un discurso, y este discurso es el signo objetivo de la reivindicación de su derecho a ser habladas y dichas públicamente (y poéticamente).

En el análisis que proponemos resulta fundamental el concepto de campo desarrollado por el sociólogo francés Pierre Bourdieu (1997). Dicho concepto hace referencia a un espacio social, un microcosmos con una autonomía relativa y poseedor de su propia lógica. En un campo determinado se producen, entre los agentes o grupos de agentes que actúan en él, enfrentamientos que responden a relaciones de fuerza. Esta fuerza es el capital simbólico acumulado y lo que está en juego es la transformación de estas relaciones de fuerza, la imposición considerada legítima de principios de visión y de división del mundo social; en resumidas cuentas, la lucha por cambiar el peso de los capitales dentro del campo, por cambiar o mantener el orden social establecido.

El campo literario presenta toda suerte de homologías (es decir, parecidos en la diferencia) estructurales y funcionales con el campo social en su conjunto. Como él (y de la misma manera que el campo político, cuyas relaciones se encuentran alteradas bajo el franquismo) tiene sus dominantes y sus dominados, sus conservadores y su vanguardia, sus luchas subversivas y sus mecanismos de reproducción, siempre teniendo en cuenta que cada uno de esos fenómenos reviste en su seno una forma completamente específica (se trata, como hemos señalado, de rasgos estructuralmente equivalentes, lo que no quiere decir idénticos, en conjuntos diferentes). En el caso de la poesía española bajo el franquismo, que es el objeto que nos ocupa, todas estas relaciones se encuentran alteradas y determinadas por el régimen dictatorial bajo el que se desarrollan. De este modo, la conceptualización de la poesía de posguerra tendrá que hacerse inevitablemente en su relación con el campo político (dando cuenta de la sobredeterminación que este ejerce sobre aquella, así como de la pluralidad ideológica presente en el campo literario del momento). 
La aparición de Mujer sin Edén de Carmen Conde, en 1947, ayuda a situarse en los conflictos que dificultan la inserción de las poetas en una tradición y un lenguaje dados, así como en los condicionamientos que determinan el campo literario bajo la dictadura franquista.

Mujer sin Edén se constituye como un auténtico poemario inaugural que logró permear "el imaginario poético de autoría femenina en los años siguientes, dejando su rastro en la obra de otras autoras. De ahí en adelante, las variaciones en torno al mito de Eva ordenan un discurso transgresor en relación a las estructuras patriarcales, inaugurando un diálogo poético centrado en la situación del colectivo femenino" (Payeras Grau 2009: 318).

Una de las condiciones de posibilidad de esta relevancia viene dada por la posición de Carmen Conde en el campo literario del momento (es decir, por su capital simbólico, muy alejado del de muchos poetas hombres pero que no poseía en ese momento ninguna otra mujer), que se transfiere a su poemario y a la posterior cadena de diálogos y que, en gran medida, la posibilita. Lo mismo podría decirse de la antología presentada por Conde en 1954, Poesía femenina española viviente, que supuso una transgresión simbólica importante, especialmente en los años en los que se publicó. Su relevancia está determinada también por la posición de Conde en la vida cultural de esos años, que se transfiere a la antología y, de nuevo, la posibilita y la legitima. Tras ese primer volumen antológico, publicará otros dos: Poesía femenina española: 1939-1950 (1967) y Poesía femenina española: 1950-1971 (1971).

Recordemos que el acceso a la vida intelectual en la España franquista, en particular en los primeros años, suponía situarse respecto de una tradición próxima muy exigente: la que culminó en la Segunda República y que la Guerra Civil canceló institucionalmente pero no, aún, intelectualmente. No hay más que pensar en la trayectoria de Carmen Conde: había iniciado su carrera literaria antes de la guerra y gozaba ya entonces de una proyección importante; recordemos, por ejemplo, que en 1934 su libro Júbilos fue prologado por Gabriela Mistral e ilustrado por Norah Borges. No es casualidad que la persona que hace la primera antología de mujeres poetas que se publica bajo el franquismo y que inicia esta cadena de reescrituras de mitos clásicos femeninos sea una autora que inicia su socialización dentro del campo literario en la época de la República.

Por todo ello, estas antologías de poesía femenina publicadas por Carmen Conde tuvieron un significado simbólico fundamental, a pesar de los problemas que ha señalado un sector importante de la crítica (Payeras Grau, Senís Fernández) acerca del peligro de que el estudio separado y en bloque de las mujeres poetas dificulte su inserción en la historia de la literatura o construya una especie de canon 'a la contra'. El problema, en definitiva, viene dado por la asimetría de considerar antologías de género a las que incluyen solamente a mujeres, mientras que aquellas que dan cabida solamente a hombres se presentan como generales. La justificación está en la idea de mérito: en las segundas ese 
criterio, el del mérito, es el único que se declara, mientras que en las femeninas se hace explícito que el género es un criterio de exclusión.

Estas antologías, en el momento en el que se realizaron y publicaron (especialmente la primera), poseen el valor añadido de todos los movimientos de contestación del orden simbólico: contribuyen a desbaratar las evidencias. Si estas formas de contestación molestan tanto a las élites culturales es porque van en contra tanto de las disposiciones profundas como de los intereses específicos de los que podríamos llamar hombres de aparato (es decir, que ocupan un lugar central y duradero en grupos y posiciones de poder). De ahí viene su insistencia en dejar fuera de la reflexión cultural (y política) espacios enteros del mundo social (como todo lo que tiene que ver con las mujeres: arte o literatura producidos por ellas, trabajo femenino...). En el caso de estas antologías de mujeres poetas estamos, por tanto, ante la introducción en la cultura necesariamente política de muchos ámbitos que la cultura de ese momento excluía.

Una afirmación parecida podría hacerse de Mujer sin Edén y la fructífera cadena de respuestas que generó: pensemos, por ejemplo, en Mujer de barro de Ángela Figuera, Eva en el tiempo de María Beneyto o Esa mujer que soy de Susana March, por dar solamente tres nombres de primer nivel.

En Mujer sin Edén, Carmen Conde revisa a partir de la figura de Eva los mitos constitutivos del género femenino en el mundo social y cultural. Tras ser silenciadas en los textos sagrados, son las mujeres quienes toman la palabra en este largo poema para contar su propia versión de la historia (y de la Historia) y mostrar su desacuerdo con los valores que les fueron impuestos. El libro constituye, en lo fundamental, una reflexión acerca del papel de las mujeres a través de los tiempos; esa reflexión, como hemos señalado, se inicia en el mito del Génesis y se centra en el arquetipo fundacional de Eva, aunque se ramifica en otras figuras femeninas de la Biblia (Sara, María, María Magdalena...), que completan el mapa de una voz colectiva que representa a las mujeres en su conjunto y que confronta sistemáticamente su propio discurso con el discurso masculino y patriarcal, quedando de esta forma de manifiesto el sometimiento y la marginación que las mujeres han sufrido a lo largo de la Historia.

Como ha señalado Payeras Grau, "la argumentación de Carmen Conde se organiza alrededor de los siguientes asuntos: el doble rasero que el patriarcado aplica a la conducta femenina; la polaridad en la representación tradicional de la mujer; la naturaleza y la responsabilidad de la transgresión, y, por último, la denuncia de la marginación histórica de la mujer" (Payeras Grau 2009: 311).

En este sentido, resulta particularmente interesante el alegato que Conde pone en boca de Eva acerca de la cuestión de la culpa en el pecado original, especialmente en lo referido a la responsabilidad de la propia Eva en ello y a la desproporción entre la falta cometida y el castigo recibido (castigo que, como es sabido, se constituye como un pecado de linaje, por lo que alcanza a toda su descendencia). 
La representación patriarcal de la figura de Eva, responsable de la caída y la pérdida del paraíso de todos los seres humanos, lleva a Conde a organizar un discurso cuidadosamente montado sobre la síntesis de diversos episodios del Antiguo Testamento donde pone de relieve ese doble rasero que se aplica a la conducta de las mujeres. Así, por ejemplo, en el poema "La mujer no comprende" identifica las experiencias misóginas de distintos personajes bíblicos femeninos como una tragedia común para todo el colectivo de las mujeres, recurriendo a un yo poético en primera persona que se identifica con un sujeto femenino plural:

¡Cuántas veces a estéril has condenado mi vientre! ¿por qué luego que otra, Agar la egipcia pariera, hiciste que mi entraña doblara su existencia?

¿Por qué Abraham fue cobarde, por qué Isaac fue cobarde?

¿Por qué los dos dijeron: "Es mi hermana”, de mí?

¡Ay Señor, cuánto duelo en mi cuerpo permites,

dejándome sufrir sin piedad de mi enojo! (Conde 1979: 406)

La cadena de reescrituras iniciada por Mujer sin Edén resultó especialmente fructífera en el caso de la poeta Ángela Figuera, cuyo primer libro, Mujer de barro, además de ser uno de los grandes poemarios de la posguerra española, está escrito en gran medida en diálogo con el de Conde.

En Mujer de barro, publicado en 1948, la mujer está hecha del mismo material que el hombre y no de su costilla, como un apéndice del varón. Además, impugna los barrotes en los que siempre han sido encerradas las mujeres en el mundo cultural:

¡Cuán vanamente, cuán ligeramente me llamaron poetas, flor, perfume!...

Flor, no: florezco. Exhalo sin mudarme.

Me entregan la simiente: doy el fruto.

El agua corre en mí: no soy el agua. (Figuera Aymerich 2009: 32)

Las metáforas biológicas son constantes, tanto referidas a la maternidad como a la producción de la propia obra. Aunque, como vemos en el poema anteriormente citado, con frecuencia aparece la idea de que el principio generador de vida (la simiente, en palabras de Figuera) es masculino, no podemos decir que la visión de los procesos (típicamente femeninos) de gestación y parto coincida con el discurso tradicional defendido por el régimen de Franco. Frente a esa perspectiva, que plantea esos procesos como pasivos, el yo poético de Mujer de barro se vive como un sujeto completamente activo que propone una visión gozosa de la maternidad y la crianza. 
El valor que tanto Conde como Figuera otorgan en estos poemarios a la maternidad - no solamente en la vida de las mujeres sino como principio vertebrador y pivote sobre el cual tiene que girar el papel y la presencia de las mujeres como sujetos de la Historia y como redentoras de la humanidad en su conjunto- permanece (quizá de manera aún más clara en el caso de Conde que en el de Figuera) dentro de un planteamiento claramente esencialista, aunque, como hemos señalado, no se ajusta en absoluto al discurso franquista dominante. Teniendo en cuenta que estamos hablando de la España de los años 40, la reflexión desplegada por ambas se trataba, sin duda, de un discurso difícil de conquistar.

Pocos años después, Ángela Figuera desarrollará un pensamiento mucho más crítico y más cercano a la poesía social en lo que respecta al discurso sobre la maternidad. En El grito inútil, de 1952, desmitifica dicha experiencia y cada una de sus etapas, desde la relación sexual hasta la crianza de los hijos, será contada sin vanas euforias. En este poemario encontramos, por ejemplo, el famoso "Mujeres del mercado", que finaliza diciendo:

Van a un patio con moscas. Con chiquillos y perros.

Con vecinas que riñen. A un fogón pestilente.

A un barreño de ropa por lavar. A un marido con olor a aguardiente y a sudor y a colilla.

Que mastica en silencio. Que blasfema y escupe.

Que tal vez por la noche, en la fétida alcoba, sin caricias ni halagos, con brutal impaciencia de animal instintivo, les castigue la entraña con el peso agobiante de otro mísero fruto.

Otro largo cansancio. (Figuera Aymerich 2003: 142-143)

Esta doble réplica de género aparece a cada paso en la obra de Ángela Figuera. Si pensamos en sus libros poéticos a partir de Vencida por el ángel, veremos que, como otras poetas sociales, escribe sobre los mismos temas que los hombres (que tienen la consideración de generales), y además de los relativos a la vida de las mujeres, cuyas experiencias estaban invisibilizadas. Como es habitual, lo masculino aparecía en el discurso dominante como lo neutro, lo no marcado, y la única marca de género que se percibe es la femenina.

Lo que las mujeres han recorrido, nos dice Figuera en sus poemarios, no es solamente la historia de los hombres, al igual que ellos, sino su propia opresión específica: una historia, tal como nos relata la autora, de una violencia inaudita. De ahí que se detenga en sus textos en una maternidad no idealizada, en la vida doméstica, las relaciones conyugales, así como en otras experiencias que formaban parte del día a día de millones de mujeres, como ir al mercado en 
condiciones económicas precarias, o que construya metáforas de la sangre alusivas al parto o a la menstruación (por ejemplo, "La sangre", Figuera Aymerich 2009: 205-206). Es por eso por lo que estos discursos poéticos son, en cierto modo, un trabajo de teoría crítica, al ser textos de contestación con voluntad de ejercer algún efecto sobre la realidad. Naturalmente, se trataba de poemas que llegaban a un público escaso (como ocurría con la poesía en general y con la escrita por mujeres en particular), pero la crítica siempre tiene límites que vienen dados por las condiciones objetivas en las que se desarrolla.

El discurso crítico de Figuera (como el de otras autoras del momento) tiene, como decíamos al inicio de este trabajo, otra consecuencia fundamental: supone una redefinición de la experiencia poética y de los aspectos de la realidad susceptibles de ser poetizadas. Estos discursos son, como señalábamos, el signo objetivo de la reivindicación de su derecho a ser habladas y dichas públicamente (y poéticamente).

Como correlato necesario al mito de Eva, dibujada en estos poemarios fundamentalmente como figura materna, tanto Conde como Figuera dedican muchas reflexiones a los mitos de Caín y Abel, en unos términos que, tratándose de dos poetas españolas en la inmediata posguerra, no resulta difícil interpretar como una forma de aludir, sin hacerlo directamente, a la Guerra Civil. Se trata de uno de los ejemplos más claros de un mecanismo que funciona también en el discurso con perspectiva de género desarrollado no solamente por ambas autoras sino por el resto de poetas que, bajo el franquismo, conforman el diálogo e intercambio poético del que venimos hablando: la reescritura de mitos clásicos, como de figuras arquetípicas, era un recurso efectivo para eludir la censura en la España de Franco.

En las producciones culturales se superponen siempre diferentes juegos de lenguaje: lenguajes teóricos, políticos, estéticos... El trabajo sociológico es una de las maneras de analizarlos y desenredarlos. Se trata del mismo fenómeno que Bourdieu destacaba a propósito de Heidegger y que puede resultar útil para pensar otras producciones culturales. El pensamiento filosófico, decía Bourdieu, es, en mayor o menor medida, un pensamiento bizco (Bourdieu 1988: 9-14) en el que se dice una cosa sin dejar de aludir a otra por el modo de decirlo y por los signos con los que se siembra la expresión. Si a todo eso añadimos la situación política y el lugar que se ocupe en el campo literario, esa cuestión de la producción bizca, que mira con un ojo hacia la obra sin dejar de mirar con el otro a muchas realidades que la determinan, se multiplica hasta casi el infinito en una situación de heteronomía como la de la producción literaria en la posguerra española. Y más si se es una mujer.

De este modo, el discurso poético, como cualquier otra forma de expresión en los años de los que hablamos, es resultado de una transacción entre una intención expresiva y la censura ejercida por el universo político y social en el que se produce. Así, para comprender la poesía española de posguerra en su lógica 
inseparablemente literaria y política, hay que rehacer el trabajo de dobles sentidos que le permite desarrollarse. Hay que analizar la lógica de doble sentido y de sobreentendido (sobre todo si nos situamos en la parte izquierda del campo) que permite a las palabras (o a los mitos o arquetipos) funcionar simultáneamente dentro de dos registros hábilmente unidos y separados. En ocasiones, los propios autores hacen explícito el procedimiento, en una reflexión metaliteraria sobre ese sentido doble y esa constante alusión/elusión: no hay más que recordar, por ejemplo, el poema "Biotz-begietan" de Blas de Otero, con su repetido "Escribo y callo", o "No quiero" de Ángela Figuera.

Si esa lógica del doble sentido se da en todos los discursos críticos con el régimen, sucede en mayor medida si se es una mujer, que es una variable fundamental al estudiar ese lugar en el que están situadas las personas que producen las obras culturales y que determinan su valor. Ser mujer es una marca simbólica negativa que además se retroalimenta (negativamente) cuando escribe textos sobre cuestiones que afectaban a la vida de las mujeres.

Así, la trayectoria de un o una poeta, como de cualquier otro productor cultural, comprende, entre otras, dos condiciones importantes: la adquisición de un determinado capital cultural y la inversión del mismo en la comunicación pública. Los distintos tipos de capital cultural y los modos de inversión del mismo determinan un mayor o menor reconocimiento, siempre que tales actividades se acompasen bien con los modelos intelectuales dominantes. En cuanto al tipo de capital cultural, existen recursos culturales que permiten o no participar en los debates centrales de una coyuntura intelectual concreta. La transmisión posibilita la adquisición de unos recursos culturales compartidos, al menos en teoría, por un conjunto de individuos susceptibles de entrar en debate. Pero la transmisión de ciertos contenidos culturales también puede condenar tanto a sus poseedores como a sus receptores a la marginalidad intelectual. Es lo que ocurre con la poesía escrita por mujeres, que se constituye casi como una especie de subcampo (también en lo relativo a su consideración cultural peyorativa, que no es más que un juicio social denegado). Tanto si se escribe dentro las características de la supuesta poesía de lo femenino (sometida a esa irreductible esencia, la feminidad), como si, tal como ocurre en el caso de la reescritura de mitos clásicos, el texto ejerce una crítica sobre el presente (acompasándose así, teóricamente, con la línea poética dominante, la de la poesía social), el discurso quedará relegado a la marginalidad intelectual: hablar del parto, de la menstruación o de la dureza de la vida de las mujeres de clases desfavorecidas no era una opción legítima en el mercado de los bienes simbólicos.

Conviene, para analizar esta problemática, diferenciar entre aparato y campo. Dicha distinción la establece Bourdieu (1997) y posteriormente se detiene en ella Gisèle Sapiro (2007). Bourdieu reserva el término aparato a aquellos casos en los que, en un campo, los dominantes tienen los medios para anular la 
resistencia y la reacción de los dominados. No obstante, según Sapiro, esa definición es demasiado extrema, ya que, dice esta socióloga, incluso en los regímenes más autoritarios y que emplean los métodos de control más sofisticados, siempre existe una resistencia, aunque sea en la clandestinidad. Además, y creo que esto es aún más importante para nuestro tema, defiende Sapiro que siempre que hay un campo literario o intelectual ya constituido subsiste una forma de autonomía. De hecho, en España es eso lo que hace que el campo literario, sobre todo en lo que respecta a la poesía, y el político estén desincronizados, ya que las líneas poéticas centrales de los años 40 y 50 desarrollan disposiciones críticas hacia el régimen.

Entre los medios de control que apunta Sapiro está la autorización de imprimir, algo que el mercado había ido eliminando en la práctica ya en el siglo XIX pero que en España revitalizó el franquismo (significativamente, en latín: imprimatur, nibil obstat). Simultáneamente, los campos culturales se llenaron de estrategias diversas para eludir la presión: uso de códigos y alusiones veladas, publicaciones en el extranjero o clandestinas, desplazamientos en el tiempo y en el espacio geográfico o, como hemos dicho, en el caso de la poesía escrita por mujeres, uso de mitos o arquetipos. Es importante, en este sentido, destacar que el combate contra el control político de la producción cultural, contra la heteronomía, ha contribuido a fundar los principales valores sobre los que reposa la autonomía relativa del campo literario, incluso si, como ocurre en las dictaduras o los regímenes totalitarios, la defensa de la autonomía está asociada a una lucha política a la que la primera, la autonomía, queda subordinada.

Así, obras como Mujer sin Edén o Mujer de barro son instrumentos políticos muy potentes aunque no hablen directamente de política. Ello es así porque, como antes comentábamos, van en contra de las disposiciones profundas y los intereses específicos de las personas y grupos que ocupan los lugares centrales (en este caso, tanto del campo político como del literario, copado por hombres). Estamos, como decíamos, ante la introducción en la cultura de muchos ámbitos (en la problemática que nos ocupa, relativos a la vida de las mujeres) que la definición de cultura de ese momento excluía. Y ese acto es, inevitablemente, político.

Hay un poema de Ángela Figuera, significativamente no recogido en ningún libro, que se llama "A Carmen Conde, mujer sin edén”. En él, Figuera interpela a Conde, identificándola con su Eva de Mujer sin Edén, y a Eva con todas las mujeres del mundo que buscan una humanidad nueva y una vida social libre de injusticias y discriminación. De ahí surge la imagen de una mujer (la madre Eva, la madre Conde) cuya nostalgia del paraíso perdido se suma al dolor de la guerra fratricida. La rigurosa reflexión de Figuera desarma, de manera explícita en la última estrofa, el discurso misógino y patriarcal. Recuperando, en la misma línea de Conde, el tópico sobre la culpabilidad de la caída, Figuera ironiza en un amargo verso final: 
Tú, Carmen Conde, sabes qué sepultados ojos acechan horizontes del misterio celeste.

Tú sabes cómo el plomo pesa sobre la nube y qué sucia cortina de telarañas cierra las trémulas gargantas en profético trance. Tú sabes que, a despecho de los lúcidos raptos, setenta veces siete puertas sin cerradura custodian el recinto de la Verdad. Y cantas.

Porque tú, desterrada del Jardín, sacudida por la lluvia y el cierzo, calcinada por soles implacables, doblada por antiguos cansancios, con tus dos pies desnudos sobre piedras hostiles, con tus manos ligadas por remotos decretos, tenazmente deslindas tus caminos y buscas aquel rayo sin sombra que brilló en el principio.

(Oh nostalgia del limpio Paraíso, del Hombre recién hecho que hallaste respirando a tu lado cuando flores y bestias se te daban sumisas. $\mathrm{Y}$ tus hijos, tus únicos, tus auténticos hijos, Caín y Abel doliéndote como dos llagas tórpidas en la férvida carne.)

Tú, mujer en exilio, sumergida en mareas seculares y amargas, no renuncias. Inquieres. Tú, vencida, disuelta, resurrecta, juzgada, clamas alto con grito de agudísimo vuelo por tu amor, tu pecado, tu ignorancia y tu sino.

Porque Eva no sabía. La Serpiente sabía. Dios sabía y callaba consintiendo. La fuerza del Varón no detuvo ni cortó aquella mano.

Y la culpa fue nuestra. Nuestra culpa. Eso dicen. (Figuera 2009: 336-337)

Hay otro poema de Ángela Figuera que resulta significativo en lo relativo a esa especie de hermandad (casi al modo de las poetas románticas) entre poetas españolas de posguerra. Está dedicado, como reconocimiento de su poemario fundacional, a Carmen Conde y aparece una nueva referencia a Eva como arquetipo femenino originario pero, de acuerdo con la lógica de estas reescrituras, sus características aparecen no solamente como positivas sino como ejemplares. El poema se publicó en la revista Espadaña en 1950 pero, de nuevo, no se recogió en libro. Se trata de "Exhortación impertinente a mis hermanas poetisas" (Figuera 2009: 308-309): 
Porque, amigas, os pasa que os halláis en la vida como en una visita de cumplido. Sentadas cautamente en el borde de la silla. Modosas. Dibujando sonrisas desvaídas. Lanzando suspirillos rimados, como pájaros bobos. [...]

Levantaos, hermanas. Desnudaos la túnica. Dad al viento el cabello. Requemaos la carne con el fuego y la escarcha de los días violentos y las noches hostiles aguzadas de enigmas.

[...]

Eva quiso morder en la fruta. Mordedla. Y cantad el destino de su largo linaje dolorido y glorioso. Porque, amigas, la vida es así: todo eso que os aturde y asusta.

Dos son los criterios (aunque él mismo admite que podrían ser más) que Louis Pinto (1998: 15-16) propone para esclarecer la calidad de una producción intelectual y que nos pueden resultar de utilidad; el primero, un criterio polémico: ¿propone un punto de vista nuevo que en el estado dominante de los saberes era difícil conquistar? Y, en segundo lugar, un criterio económico: ¿permite este punto de vista una extensión del conocimiento a través de las generalizaciones y comparaciones que su puesta en funcionamiento provoca?

Si referimos ambas cuestiones a la reescritura de mitos clásicos llevados a cabo por las poetas de posguerra, la respuesta a ambas preguntas, tal como venimos viendo, sería un rotundo sí.

Mujer sin Edén y Mujer de barro no solamente influyeron en sus coetáneas, que hicieron suya esa elocuencia para hablar de su condición presente, sino que inauguró una línea ininterrumpida hasta hoy de reinterpretación de mitos clásicos por parte de las mujeres poetas para denunciar la discriminación que sufren las mujeres por el hecho de serlo.

Así, en España, a partir de los años ochenta, la consolidación de la democracia y la mayor presencia de mujeres en poesía van a permitir nuevas lecturas que muestran construcciones hasta ese momento inéditas de activos sujetos femeninos que contestan de manera radical al ideario tradicional de la mujer como objeto y en particular como objeto de la literatura. Con frecuencia, ese nuevo sujeto activo toma la forma de un sujeto erótico que, con un erotismo lúdico, utiliza subversión, la ironía y la parodia para encontrar un nuevo modo de decir el cuerpo, la sexualidad y los sentimientos femeninos. Dentro de ese objetivo, aparece con frecuencia la revisión y subversión de mitos clásicos (pensemos, por ejemplo, en Ana Rossetti).

Para finalizar, conviene insistir en la importancia de las revisiones y subversiones de mitos clásicos realizados por estas escritoras como una forma 
de compromiso y de contestación a la doxa porque contribuyeron a construir posibilidades o, mejor dicho, condiciones de posibilidad en las formas de decir y por tanto también de vivir, a pesar de las limitaciones, de todo tipo y desde todos los campos, con las que se toparon. En ese sentido, contribuyeron a posibilitar la existencia real de las mujeres como agentes históricos en las prácticas culturales y la literatura. 
El linaje de Eva: revisiones de mitos clásicos: en las poetas españolas bajo el franquismo

\section{Bibliografía}

Bourdieu, Pierre (1988). L'ontologie politique de Martin Heidegger. París: Les Éditions de Minuit.

(1997). Las reglas del arte. Génesis y estructura del campo literario. Barcelona: Anagrama.

Conde, Carmen (1954). Poesía femenina española viviente: antología. Madrid: Arquero.

(1967). Poesía femenina española: 1939-1950. Barcelona: Bruguera.

(1971). Poesía femenina española: 1950-1960. Barcelona: Bruguera.

(1979). Obra poética 1929-1966. Madrid: Biblioteca Nueva.

Figuera Aymerich, Ángela (2009). Obras Completas. Madrid: Hiperión.

Payeras Grau, María (2003). Espejos de palabra. La voz secreta de la mujer en la poesía española de posguerra (1939-1959). Madrid: UNED.

Pinto, Louis (1998). Pierre Bourdieu y la teoría del mundo social. México D.F.: Siglo XXI Editores.

Rossetti, Anna (2004). La Ordenación (Retrospectiva 1980-2004). Sevilla: Fundación José Manuel Lara.

Sapiro, Gisèle (2007). "Pour une approche sociologique des relations entre littérature et idéologie", COnTEXTES, núm. 2 [http://contextes.revues. org/165;DOI:10.4000/contextes.165]

Senís Fernández, Juan (2004). "Canon a la contra y antologías en la última poesía española escrita por mujeres”, Clarin, 52: 10-14. 